\title{
Editorial
}

\section{Completely Monotonic and Related Functions: Their Applications}

\author{
Senlin Guo, ${ }^{1}$ Andrea Laforgia, ${ }^{2}$ Necdet Batir, ${ }^{3}$ and Qiu-Ming Luo ${ }^{4}$ \\ ${ }^{1}$ Department of Mathematics, Zhongyuan University of Technology, Zhengzhou, Henan 450007, China \\ ${ }^{2}$ Department of Mathematics, Roma Tre University, Largo San Leonardo Murialdo 1, 00146 Rome, Italy \\ ${ }^{3}$ Department of Mathematics, Faculty of Arts and Sciences, Nevsehir University, 50300 Nevsehir, Turkey \\ ${ }^{4}$ Department of Mathematics, Chongqing Normal University, Chongqing 401331, China
}

Correspondence should be addressed to Senlin Guo; sguo@hotmail.com

Received 1 October 2014; Accepted 1 October 2014; Published 31 December 2014

Copyright (C) 2014 Senlin Guo et al. This is an open access article distributed under the Creative Commons Attribution License, which permits unrestricted use, distribution, and reproduction in any medium, provided the original work is properly cited.

Completely monotonic and related functions are important function classes in mathematical analysis. It was Bernstein [1] who in 1914 first introduced the notion of completely monotonic function. This year we celebrate its 100th anniversary. In 1921, Hausdorff [2] gave the notion of completely monotonic sequence, which is related to the notion of completely monotonic function. Widder [3] in 1931 introduced the notion of minimal completely monotonic sequence. The terminology: logarithmically completely monotonic function, was first used in [4] in 1988. In 1989, the authors [5] introduced the notion of strongly completely monotonic function. In 2009, the authors [6] introduced the notion of strongly logarithmically completely monotonic function. Also, the terminology: almost strongly completely monotonic function, was introduced in [6] in order to simplify the statement of the main results of the article [6]. In 2012, the terminology of almost completely monotonic function was defined and used in [7] in order to simplify the statement of the main results of the article [7]. Researchers did a lot of investigations, in both theory and applications, on these functions. More recently, for example, the authors [6] showed that a strongly logarithmically completely monotonic function must be almost strongly completely monotonic. Also, in [6], the following fact that a strongly logarithmically completely monotonic function cannot be strongly completely monotonic, or, equivalently, a strongly completely monotonic function cannot be strongly logarithmically completely monotonic was established. Figure 1 illustrates the inclusion relationships among these classes of functions on $(0, \infty)$. In these inclusion relationships, the fact that a logarithmically completely monotonic function must be completely monotonic was, in fact, first proved in [8] although the author did not use the terminology of logarithmically completely monotonic function; all others are from [6] or directly from their definitions. Also note that in [6] counter examples were presented to show that some function classes are not included in some other function classes or to show that the intersection sets of two function classes are not empty.

We invited investigators to contribute original research articles as well as comprehensive review articles to this special issue which will stimulate the continuing efforts to understand these functions. This special issue mainly focuses on the applications, in all fields, of completely monotonic and related functions. The topics included in this special issue are as follows:

(i) completely monotonic functions and their applications,

(ii) almost completely monotonic functions and their applications,

(iii) logarithmically completely monotonic functions and their applications,

(iv) strongly logarithmically completely monotonic functions and their applications, 


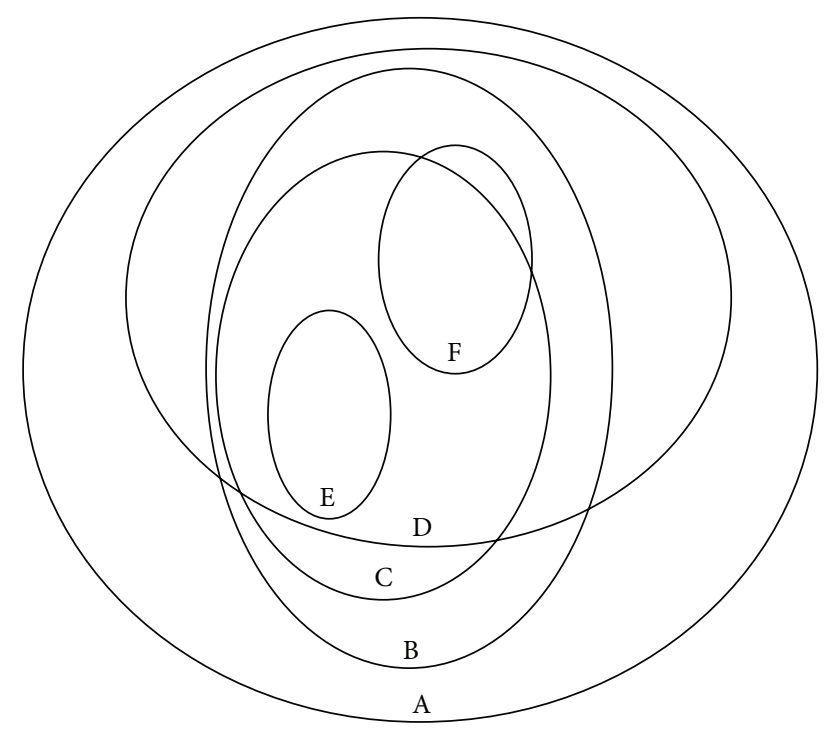

FIGURE 1: Inclusion relationships among classes of completely monotonic and related functions on $(0, \infty)$. (A) Almost completely monotonic function class. (B) Completely monotonic function class. (C) Logarithmically completely monotonic function class. (D) Almost strongly completely monotonic function class. (E) Strongly logarithmically completely monotonic function class. (F) Strongly completely monotonic function class.

(v) strongly completely monotonic functions and their applications,

(vi) almost strongly completely monotonic functions and their applications,

(vii) absolutely monotonic functions and their applications,

(viii) Bernstein functions and their applications,

(ix) completely convex functions and their applications,

(x) moment sequences and their applications,

(xi) Laplace (Stieltjes) transforms associated with completely monotonic or related functions,

(xii) interpolation or fitting of data by completely monotonic or related functions,

(xiii) approximation of (by) completely monotonic or related functions,

(xiv) analytic inequalities associated with completely monotonic or related functions,

(xv) numerical methods for completely monotonic or related functions, explicit and implicit,

(xvi) ordinary, partial, and fractional differential equations and difference equations related to completely monotonic or related functions,

(xvii) mathematical modeling using completely monotonic or related functions.

Dozens of manuscripts were received for this special issue, but only a few peer-reviewed, high-quality articles were published. We hope that the interested readers will continue to investigate the applications of completely monotonic and related functions in probability and statistics, numerical and asymptotic analysis, statistical physics, physical chemistry, and so forth.

\section{Acknowledgments}

The guest editors would like to express their gratitude to all authors who submitted their papers for possible publication in this special issue and to the reviewers whose comments and suggestions on the manuscripts were important to them. They also would like to thank the editorial board members for their support to this special issue.

\section{Senlin Guo Andrea Laforgia \\ Necdet Batir Qiu-Ming Luo}

\section{References}

[1] S. Bernstein, "Sur la définition et les propriétés des fonctions analytiques d'une variable réelle," Mathematische Annalen, vol. 75, no. 4, pp. 449-468, 1914.

[2] F. Hausdorff, "Summationsmethoden und momentfolgen I," Mathematische Zeitschrift, vol. 9, no. 1-2, pp. 74-109, 1921.

[3] D. V. Widder, "Necessary and sufficient conditions for the representation of a function as a Laplace integral," Transactions of American Mathematical Society, vol. 33, pp. 851-892, 1931.

[4] R. D. Atanassov and U. V. Tsoukrovski, "Some properties of a class of logarithmically completely monotonic functions," Comptes Rendus de l'Academie Bulgare des Sciences, vol. 41, no. 2, pp. 21-23, 1988.

[5] S. Y. Trimble, J. Wells, and F. T. Wright, "Superadditive functions and a statistical application," SIAM Journal on Mathematical Analysis, vol. 20, no. 5, pp. 1255-1259, 1989.

[6] S. Guo and H. M. Srivastava, "A certain function class related to the class of logarithmically completely monotonic functions," Mathematical and Computer Modelling, vol. 49, no. 9-10, pp. 2073-2079, 2009.

[7] H. M. Srivastava, S. Guo, and F. Qi, "Some properties of a class of functions related to completely monotonic functions," Computers and Mathematics with Applications, vol. 64, no. 6, pp. 1649-1654, 2012.

[8] R. A. Horn, "On infinitely divisible matrices, kernels, and functions," Zeitschrift für Wahrscheinlichkeitstheorie und Verwandte Gebi ete, vol. 8, pp. 219-230, 1967. 


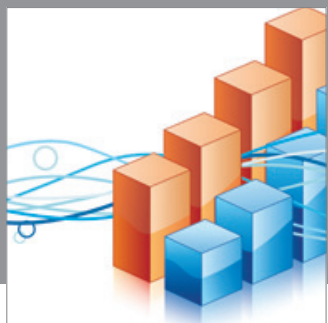

Advances in

Operations Research

mansans

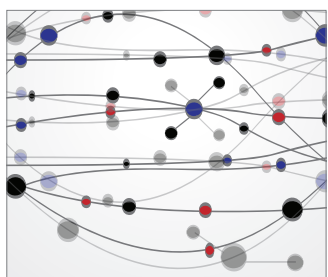

The Scientific World Journal
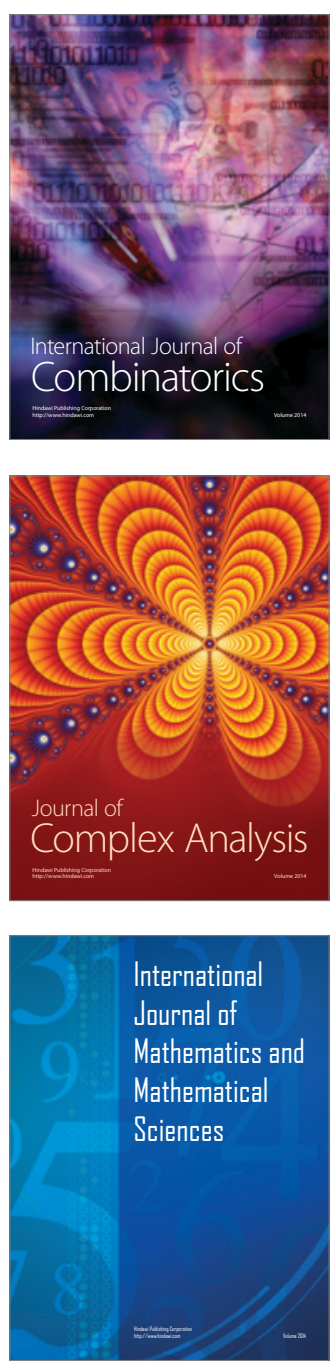
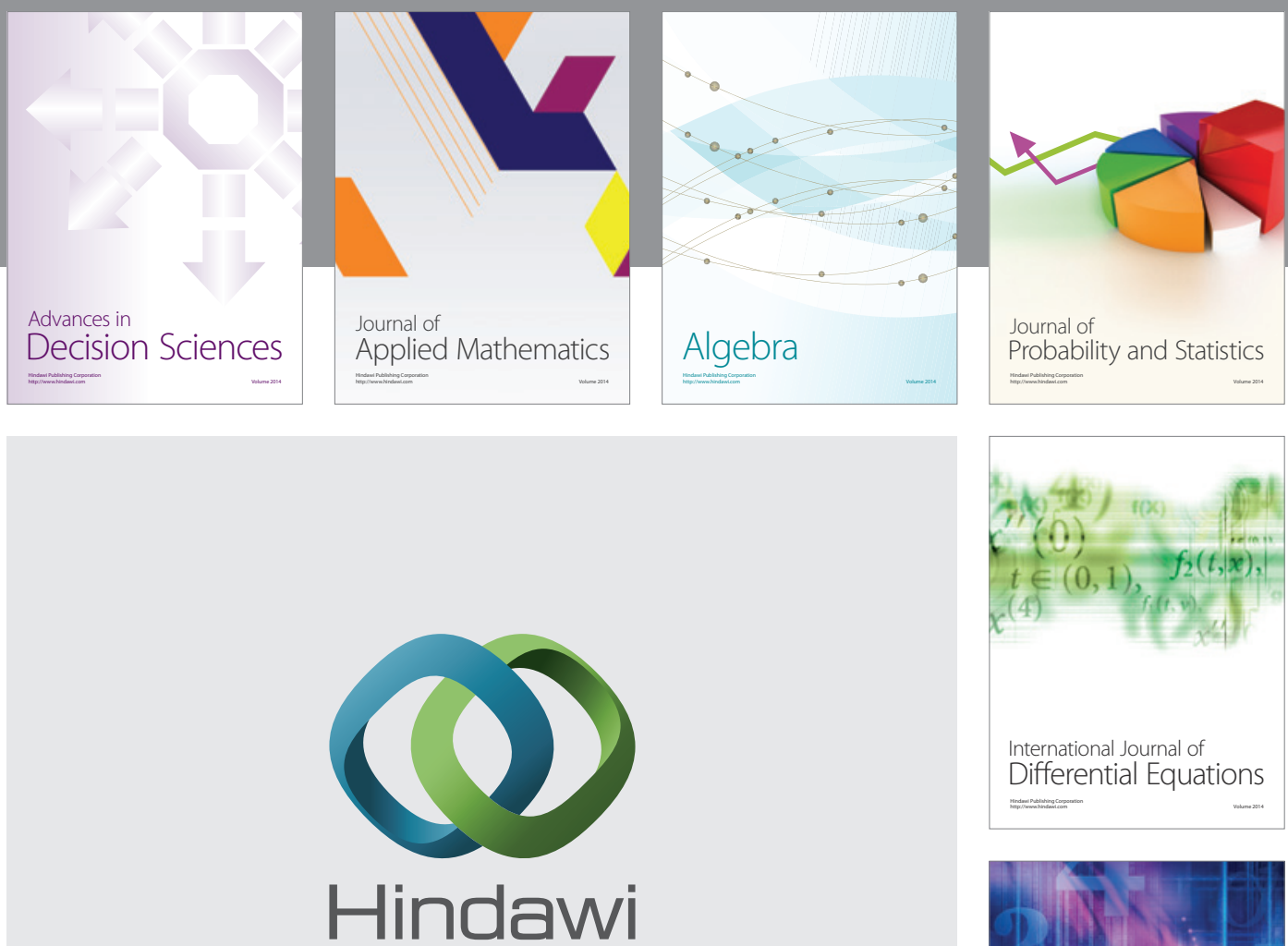

Submit your manuscripts at http://www.hindawi.com
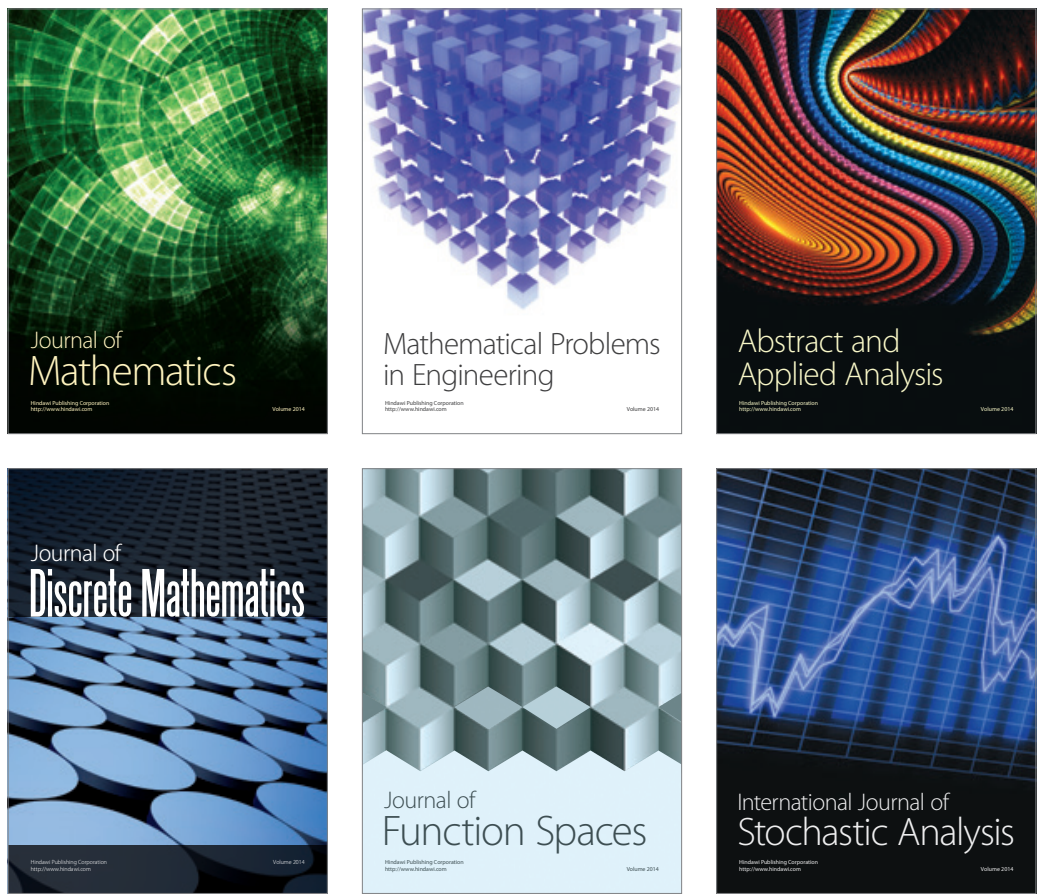

Journal of

Function Spaces

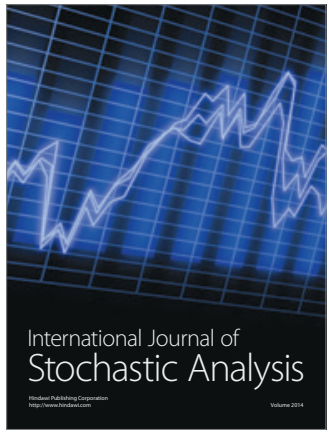

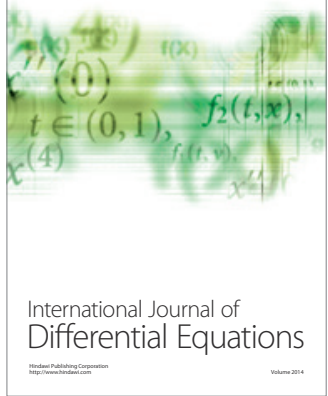
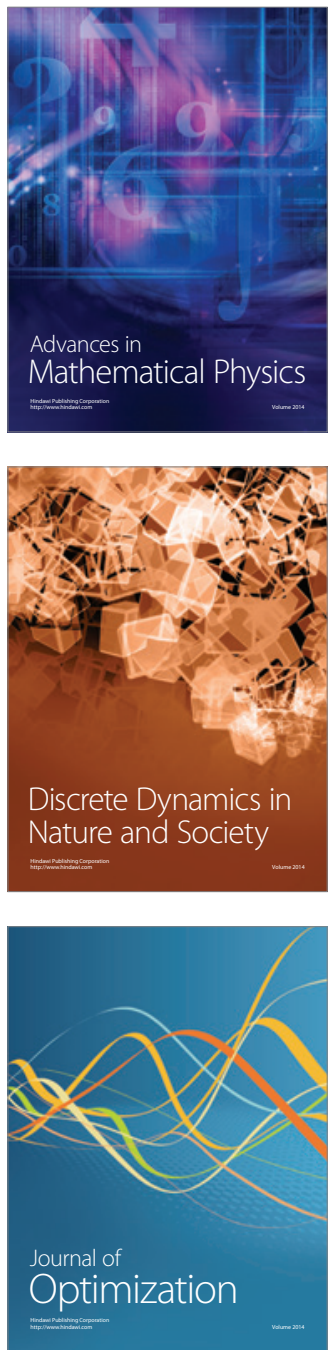